\title{
Superhard Superstrong Carbon Clathrate
}

Zihe $\mathrm{Li}^{1}$, Meng $\mathrm{Hu}^{1}$, Mengdong Ma, Yufei Gao, Bo Xu, Julong He, Dongli Yu, Yongjun Tian, and Zhisheng Zhao*

State Key Laboratory of Metastable Materials Science and Technology, Yanshan University, Qinhuangdao 066004, China

*Corresponding author. Email: zzhao@ysu.edu.cn

${ }^{1}$ These authors contributed equally 


\begin{abstract}
Carbon clathrate is a kind of typical carbon allotrope that has an open framework composed of various types of cages, displaying intriguing electronic and mechanical properties. This paper proposes a superhard superstrong carbon clathrate via first-principle calculations. This carbon clathrate, called $\mathrm{C}_{60}$ clathrate, contains 60 carbon atoms buckled through $s p^{3}$-hybridized bonds in a cubic unit cell with symmetry of $I m-3 m$, and possesses the network topology of commonly called binodal net. $\mathrm{C}_{60}$ clathrate is energetically more stable than fullerene $\mathrm{C}_{60}$ at ambient pressure, and it is more favorable than graphite at a pressure above $50.1 \mathrm{GPa} . \mathrm{C}_{60}$ clathrate can be constructed by small $\mathrm{C}_{24}$ cage and flat $\mathrm{C}_{18}$ drum. It shows a high density of $3.34 \mathrm{~g} / \mathrm{cm}^{3}$, which is the densest carbon clathrate to date. The estimated Vickers hardness and tensile strength of $\mathrm{C}_{60}$-clathrate are comparable with those of diamond, reaching remarkably high values of 91.6 and $90.7 \mathrm{GPa}$, respectively. The estimated Vickers hardness, tensile strength, and shear strength of $\mathrm{C}_{60}$ clathrate reach remarkably high values of $82.8,90.7$, and $76.4 \mathrm{GPa}$, respectively, indicating its superhard and superstrong characteristic. Band structure calculations indicate that $\mathrm{C}_{60}$ clathrate is semiconductive with a direct band gap of $2.26 \mathrm{eV} . \mathrm{C}_{60}$ clathrate can be potentially used in photovoltaic devices.
\end{abstract}




\section{Introduction}

Carbon can form a large number of allotropes with distinct electronic and mechanical properties, such as superhard insulators (diamond and lonsdaleite), ultrasoft semimetals (graphite), and superconductors (doped diamond), because of its unique capability to form $s p-, s p^{2}$-, and $s p^{3}$-hybridized bonds [1-5]. In recent years, carbon clathrates with $3 \mathrm{D} s p^{3}$-bonded networks constructed by coplanar polyhedral cages have attracted considerable interest. Benedek et al. [6] first proposed three basic series of periodic lattices of carbon clathrates, which are denoted as $f c c-\mathrm{C}_{136}, s c-\mathrm{C}_{46}$, and hex- $\mathrm{C}_{40}$. Experimentally, these clathrates have been synthesized in pure or guest atom-modified $\mathrm{Si}$ and $\mathrm{Ge}$ structures [7-13]. However, similar carbon clathrates have not been synthesized so far. Some pioneer theoretical works demonstrate that these carbon clathrates possess numerous attractive properties, such as good thermoelectric properties, promising mechanical properties, and wide band gap, for potential optoelectronic applications [14-27]. Carbon clathrates generally have low densities and hardness because of their porous structures. For example, the synthesized $3 \mathrm{D} \mathrm{C}_{60}$ polymers have a density of $2.4-2.6 \mathrm{~g} / \mathrm{cm}^{3}$ and hardness of 35-45 GPa, which are much lower than those of diamond. By regulating the size, shape, and structure of basic cage units, diverse carbon clathrates can be designed. In particular, carbon clathrates with high hardness, high strength, and certain porosity are expected.

In this study, we proposed predicted a cubic carbon clathrate with novel electronic and mechanical properties through first-principle calculations. This clathrate structure is constructed by $\mathrm{C}_{24}$ cage and flat $\mathrm{C}_{18}$ drum with 60 carbon atoms in a unit cell, and it is named $\mathrm{C}_{60}$ clathrate. $\mathrm{C}_{60}$ clathrate has a structural topology of called binodal net with the systematic name of sdt. $\mathrm{C}_{60}$ clathrate is dynamically and mechanically stable with energy lower than that of fullerene $\mathrm{C}_{60}$ at ambient pressure. Under hydrostatic compression, $\mathrm{C}_{60}$ clathrate is energetically more stable than graphite above $50.1 \mathrm{GPa}$. The density of $\mathrm{C}_{60}$ clathrate is $3.34 \mathrm{~g} / \mathrm{cm}^{3}$, which is slightly lower than that of diamond but higher 
than that of other known carbon clathrates. Electronic band structure calculations reveal that $\mathrm{C}_{60}$ clathrate is a semiconductor with a direct band gap of $2.26 \mathrm{eV} . \mathrm{C}_{60}$ clathrate is a novel superhard carbon with the estimated Vickers hardness of $82.8 \mathrm{GPa}$. Compared with diamend, $\mathrm{E}_{60}$-clathrate is a novel superhard material with Vickers hardness of $91.6 \mathrm{GPa}$-Furthermore, we systematically investigated the ideal tensile and shear strength of $\mathrm{C}_{60}$ clathrate, and the results revealed that the $\mathrm{C}_{60}$ clathrate has a tensile strength similar with that of diamond, but its shear strength is lower than diamond. Furthermere, we systematically investigated the uniaxial stress-strain relations of $\mathrm{C}_{60}$-clathrate in three principal symmetry directions.

\section{Calculation methods}

Crystal structure prediction search is based on global minimization of free energy surfaces merging $a b$ initio total-energy calculations via the particle swarm optimization (PSO) technique as implemented in the CALYPSO code [28]. First-principle calculations were carried out using the CASTEP [29] code based on density functional theory (DFT) [30, 31]. The exchange and correlation effects were described by the local density approximation (LDA) exchange-correlation functional of Ceperley and Alder, as parameterized by Perdew and Zunger (CA-PZ) [32]. Ultrasoft pseudopotentials were expanded using a plane-wave basis set with a cutoff energy of $310 \mathrm{eV}$, and a k-point spacing $\left(2 \pi \times 0.04 \AA^{-1}\right)$ was assigned to generate Monkhorst-Pack k-point grids for Brillouin zone sampling [33]. Structural optimization using the BFGS minimization method was performed until the energy change of each atom was less than $5 \times 10^{-6} \mathrm{eV}$, the forces on atoms were less than $0.01 \mathrm{eV} \AA^{-1}$, and all stress components were less than $0.02 \mathrm{GPa}$ [34]. The phonon modes of the equilibrium crystal structure obtained after structural relaxation were calculated using linear response theory [35]. The coordinates of the high symmetry points in reciprocal space for calculated phonon dispersion are $\mathrm{G}(0,0,0), \mathrm{H}(0.5$, 
$-0.5,0.5), \mathrm{N}(0,0,0.5)$, and $\mathrm{P}(0.25,0.25,0.25)$. The fixed strain method was used to assess the tensile and shear strength [36, 37].

\section{Results and discussion}

The crystal structure searches through the CALYPSO code were performed with cell size up to 30 carbon atoms at a pressure range of 0-100 GPa. Besides diamond, lonsdaleite, and previously predicted $M$-carbon, $Z$-carbon, and $W$-carbon etc. [38-43], our search structure simulations discovered predict a new cubic clathrate phase with $I m-3 m$ symmetry, which contains 60 carbon atoms in a unit cell and is named as $\mathrm{C}_{60}$ clathrate. This clathrate structure has been known in metal-organic framework (MOF) compounds with the topology of binodal net and systematic name of sdt [44]. Recently, the application of network topology approach to search for new allotropes of group 14 elements has been emphasized by Lars Öhrström and Michael O'Keeffe [45]. Nevertheless, the structure of sdt has not been considered for carbon so far, and $\mathrm{C}_{60}$ clathrate indeed presents a novel carbon allotrope.

We firstly study the structural feature of $\mathrm{C}_{60}$ clathrate. At zero pressure, the equilibrium lattice constant of $-\mathrm{a}_{60}$-clathrate unit cell of $\mathrm{C}_{60}$ clathrate is $7.1 \AA$ with two inequivalent atoms occupying the crystallographic $48 j(0$, $0.15296,0.69205)$ and $12 d(1 / 2,1 / 4,0)$ sites. $\mathrm{C}_{60}$ clathrate earben can be derived by stacking two kinds of cage-like structures (A and B), as shown in Fig. 1. Cage A (blue) contains 24 carbon atoms, and it is also the building block of previous proposed $b c c-\mathrm{C}_{6}$ and $f c c-\mathrm{C}_{32}[18,20]$. Cage $\mathrm{B}$ (yellow) is a drum-shaped structure with 18 atoms and composed of six hexagonal carbon rings. Each $\mathrm{C}_{24}$ cage is buckled with surrounding eight $\mathrm{C}_{18}$ cages through shared hexagonal faces. 

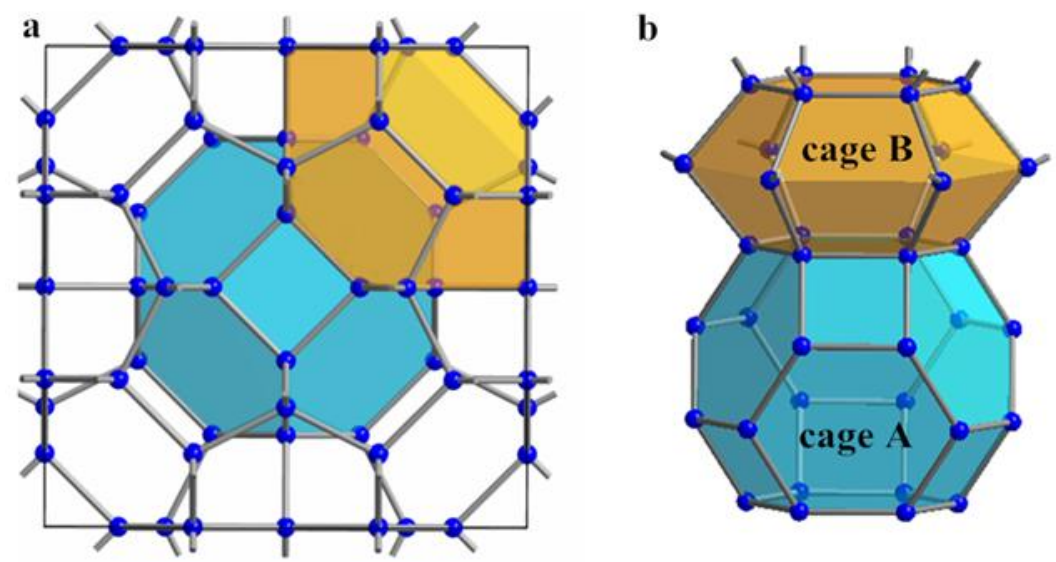

Figure 1. Crystal structure of $C_{60}$ clathrate along [100] direction (a) and the basic building blocks: cage A and cage B (b).

In comparison with other carbon clathrates, $\mathrm{C}_{60}$ clathrate has the highest density and relatively low energy (Fig. 2a). The high density is originated from the dense flat $\mathrm{C}_{18}$ drum with a much smaller volume than fullerene-like cages, such as $\mathrm{C}_{20}, \mathrm{C}_{24}, \mathrm{C}_{26}, \mathrm{C}_{28}$, and $\mathrm{C}_{60}$, which are the basic building blocks for the typical carbon clathrates such as $f c c-\mathrm{C}_{136}, s c-\mathrm{C}_{46}$, hex $-\mathrm{C}_{40}$, and $3 \mathrm{D} \mathrm{C}_{60}$ polymers (Fig. 1). As a result, this superdense carbon clathrate is expected to have high hardness and strength.

The thermodynamic stability of $\mathrm{C}_{60}$ clathrate is further examined by a direct enthalpy comparison with known experimental and theoretical carbon allotropes (Fig. 2b). $\mathrm{C}_{60}$ clathrate is metastable relative to diamend and-graphite at ambient pressure, and it has the enthalpy higher than diamond and other carbon clathrates of $f c c-\mathrm{C}_{136}, s c-\mathrm{C}_{46}$, and hex- $\mathrm{C}_{40}[6]$ at pressure from 0 to 60 GPa. However, the $\mathrm{C}_{60}$ clathrate is more stable than fullerene $\mathrm{C}_{60}$, the experimentally synthesized $3 \mathrm{D} \mathrm{C}_{60}[26,27]$, and other theoretical clathrate structures of $b c c-\mathrm{C}_{6}, s c-\mathrm{C}_{20}, f c c-\mathrm{C}_{32}$, and $\mathrm{C}_{96}$ [18-20], indicating its viability. Above $50.1 \mathrm{GPa}, \mathrm{C}_{60}$ clathrate is energetically more stable than graphite, indicating its possible synthesis from graphite under compression. $\mathrm{E}_{60}$-clathrate also has lower enthalpy than the experimentally synthesized $3 D-C_{60}$ polymer 
[26] from ambient to high pressures, which further confirms its thermodynamic stability.
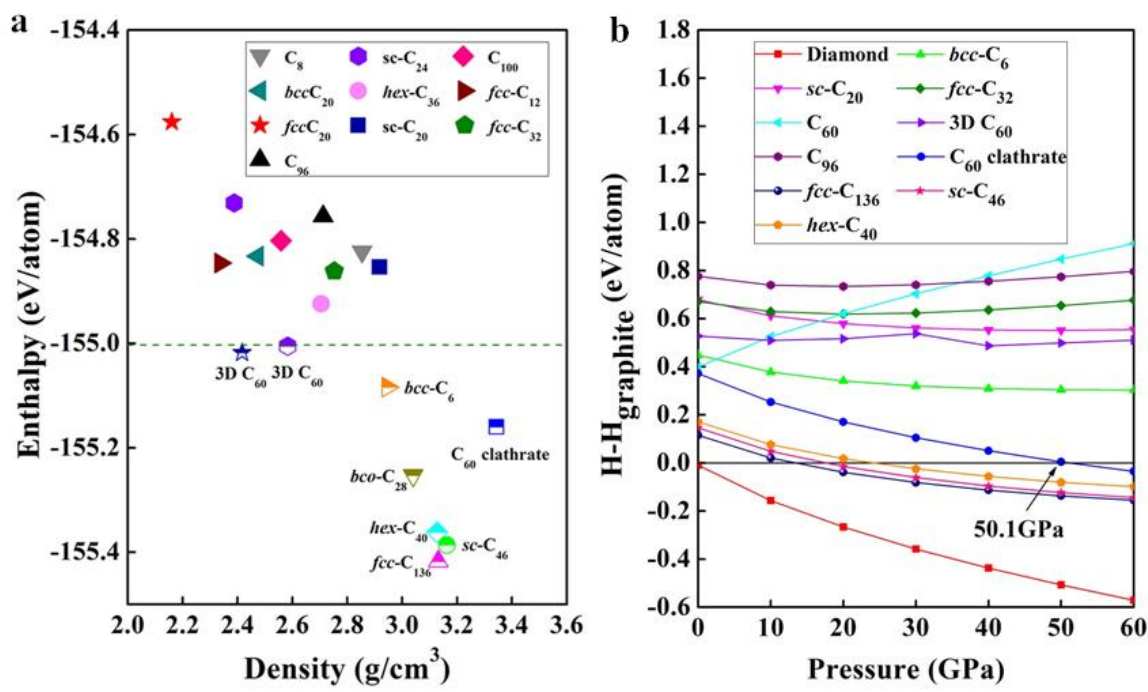

Figure 2. Enthalpy-density relationship of $\mathrm{C}_{60}$ clathrates compared with other theoretical proposed carbon clathrates and two different $3 \mathrm{D} \mathrm{C}_{60}[26,27]$ at ambient pressure (a), and enthalpy-pressure relationship of various carbon clathrates with respect to graphite (b). The dashed line is the enthalpy of experimentally synthesized $3 \mathrm{D} \quad \mathrm{C}_{60}$ polymers, presenting a partition of thermodynamic stability for carbon clathrates.

We have calculated the phonon dispersion of $\mathrm{C}_{60}$ clathrate at ambient pressure, as shown in Fig. 3a. No imaginary frequencies are observed throughout the whole Brillouin zone, suggesting the dynamic stability of $\mathrm{C}_{60}$ clathrate. The calculated highest phonon frequency of $\mathrm{C}-\mathrm{C}$ bond stretching mode in $\mathrm{C}_{60}$ clathrate is $\sim 38 \mathrm{THz}$, which is close to that of diamond (40 THz), indicating relatively strong $\mathrm{C}-\mathrm{C}$ bonding in $\mathrm{C}_{60}$ clathrate. The electronic band structure is calculated to clarify the electronic properties (Fig. 3b). $\mathrm{C}_{60}$ clathrate is a semiconductor with a direct band gap of $2.26 \mathrm{eV}$, which is about half that of indirect band gap diamond $(4.1 \mathrm{eV})$. The valence band maximum and conduction band minimum are both located at the $\mathrm{H}$ point. The direct band gap 
feature suggests it is a promising material for the solar cell or photography industry, and it can be potentially applied in light-emitting devices.
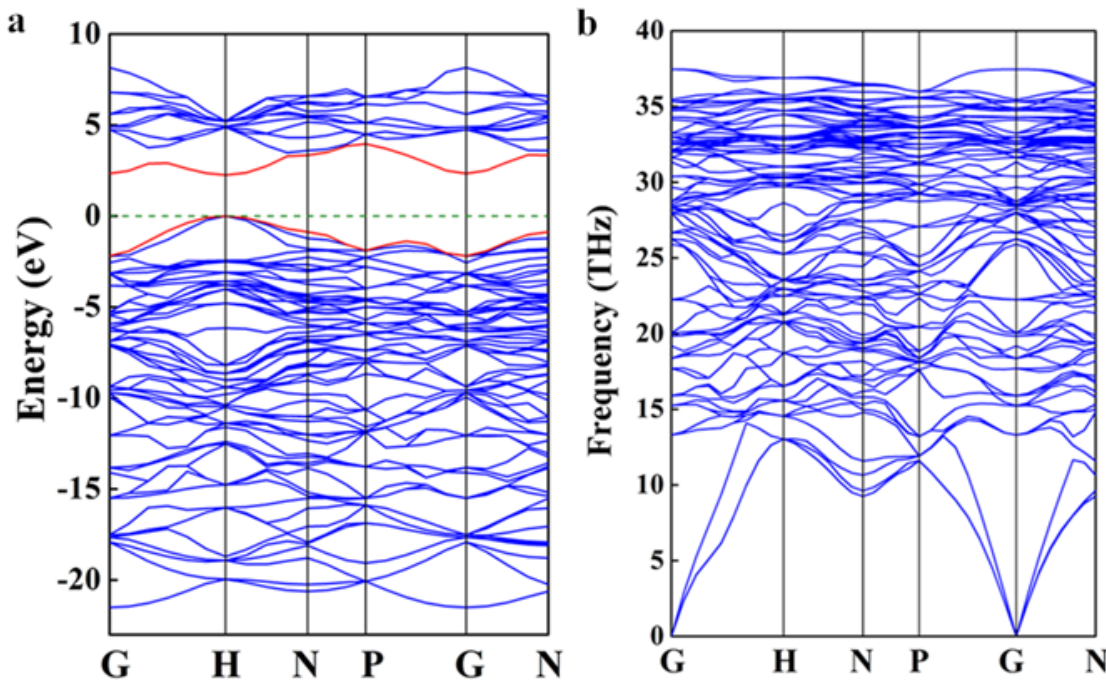

Figure 3. Electronic band structure (a) and phonon dispersion curves of $\mathrm{C}_{60}$ clathrate at ambient pressure (b).

To evaluate the mechanical stability of a crystal structure, the elastic constants of a crystal should satisfy the generalized elastic stability criteria. For a cubic crystal, the three independent elastic constants $\mathrm{C}_{11}, \mathrm{C}_{12}$, and $\mathrm{C}_{44}$ should obey the following generalized Born mechanical stability criteria: $\mathrm{C}_{11}>0, \mathrm{C}_{44}>$ $0, \mathrm{C}_{11}-\mathrm{C}_{12}>0$, and $\mathrm{C}_{11}+2 \mathrm{C}_{12}>0$ [46]. The calculated elastic constant values of $\mathrm{C}_{60}$ clathrate at ambient pressure are $\mathrm{C}_{11}=929 \mathrm{GPa}, \mathrm{C}_{44}=443 \mathrm{GPa}$, and $\mathrm{C}_{12}=123 \mathrm{GPa}$. The calculated elastic constants of $\mathrm{C}_{60}$ clathrate are positive and satisfy the Born stability criteria, indicating that $\mathrm{C}_{60}$ clathrate is mechanically stable at ambient pressure. Given that $\mathrm{C}_{60}$ clathrate possesses cubic crystal symmetry, the stiffness constant $\mathrm{C}_{11}$ can be directly related to the linear Young's modulus along crystallographic a, b, and c axes. An extremely large $\mathrm{C}_{11}(929 \mathrm{GPa})$ of $\mathrm{C}_{60}$ clathrate is observed, indicating its high incompressibility along the principal axis in the cubic $\mathrm{C}_{60}$ clathrate.

Although bulk modulus (B) and shear modulus (G) represent the resistance to material elastic deformations, they may also be associated with the fracture 
and plastic deformation of a material. Hence, the $\mathrm{B} / \mathrm{G}$ ratio can be used as a quantitative index to assess the brittle or ductile behavior of crystals. Paugh [47] proposed that a high (low) $\mathrm{B} / \mathrm{G}$ ratio is often associated with ductility (brittleness), and the critical value that differentiates ductile and brittle material is 1.75. With the Voigt-Reuss-Hill approximation [48], the bulk and shear modulus of $\mathrm{C}_{60}$ clathrate are 392 and $427 \mathrm{GPa}$, respectively. The calculated B/G ratio of $\mathrm{C}_{60}$ clathrate is 0.92 , which is bigger than that of diamond (0.82) and suggests its higher ductility than diamond. The brittleness/ductility can also be estimated by the Frantsevich rule adopting the Poisson's ratio of a material [49]. A high (low) Poisson's ratio usually suggests ductility (brittleness), and the critical value is $1 / 3$. The Poisson's ratio of $\mathrm{C}_{60}$ clathrate $(0.12)$ indicates that it is brittle, which agrees well with the estimation from the $\mathrm{B} / \mathrm{G}$ ratio.

The calculated Vickers hardness of diamond is $98.2 \mathrm{GPa}$ (in comparison with the experimental value of $96 \pm 5 \mathrm{GPa}$ ) based on the empirical formula $H_{v}(\mathrm{GPa})=350 N_{e}^{2 / 3} \mathrm{e}^{-1.191 f i} / d^{2.5}[50]$, where $N_{e}$ is the valence electron density in units of $1 / \AA^{3}, d$ is the bond length $(\AA)$, and $f_{i}$ is the Phillips ionicity of the chemical bond in a crystal [51]. The Vickers hardness $H_{4}$ - of $\mathrm{C}_{60}$-clathrate is estimated to be $91.6 \mathrm{GPa}$ and comparable with that of diamond. After the overlap populations of carbon-carbon bonds were calculated, we discovered that they are not equivalent, indicating the present of ionicity in $\mathrm{C}_{60}$ clathrate. The overlap populations of three different kinds of carbon-carbon bonds are $0.85,0.74$ and 0.84 , respectively. Therefore, we calculated the Phillips ionicity $f_{i}$ of carbon bonds in $\mathrm{C}_{60}$ clathrate by our previously established formula: $f_{i}=$ $\left(f_{h}\right)^{0.735}=\left[1-\exp \left(-\left|P_{\mathrm{c}}-P\right| / P\right)\right]^{0.735}$, where $f_{h}$ is the population ionicity scale, $P$ is the overlap population of a bond, and $P_{\mathrm{c}}$ is the overlap population of the bond in a pure covalent crystal. Here, $P_{\mathrm{c}}$ is the overlap population of diamond, and equal to 0.75 . Then, the estimated hardness of $\mathrm{C}_{60}$ clathrate is $82.8 \mathrm{GPa}$. Fig. $4 \mathrm{a}$ shows the relationship between hardness and density of $\mathrm{C}_{60}$ clathrate, $3 \mathrm{D} \mathrm{C}_{60}$ polymer [27], and some other carbon allotropes. As a result, the hardness increases with density in the carbon allotropes. This finding may provide us a 
direction to find superdense superhard carbon clathrates or other carbon allotropes. To carry out a fair consideration, we also use the macroscopic hardness model proposed by Chen et al. to estimate the hardness of carbon clathrates [52-54]. The result also shows that the four clathrates of $f c c-\mathrm{C}_{136}$, $s c-\mathrm{C}_{46}$, hex- $\mathrm{C}_{40}$, and $\mathrm{C}_{60}$ clathrate are all superhard with hardness above $70 \mathrm{GPa}$ (Fig. 4a).

Ideal strength calculations can provide valuable information on the mechanical properties of materials under stress [37,38,55-57]. In the current study, the uniaxial tensile strengths of $\mathrm{C}_{60}$ clathrate are investigated. The three primary crystal orientations, namely, [100], [110], and [111], are chosen to calculate the ideal tensile strength. The calculated tensile strengths along the [100], [110], and [111] directions are 112.4 GPa with a strain of 0.2, $90.7 \mathrm{GPa}$ with a strain of 0.21 , and $92.3 \mathrm{GPa}$ with a strain of 0.16 , respectively (Fig. 4b); these values indicate the strength anisotropy of $\mathrm{C}_{60}$ clathrate. Obviously, the lowest tensile strength is along the [110] direction, that is, the crystal will be cleaved along the [110] direction when the external stress exceeds $90.7 \mathrm{GPa}$. By contrast, the [100], [110], and [111] tensile strengths of diamond are 225, 130, and $90 \mathrm{GPa}$, respectively [57]. The lowest tensile strengths of $\mathrm{C}_{60}$ clathrate and diamond are quite close.

Slippage usually occurs in the crystal plane perpendicular to the weakest tensile direction, hence we calculated the shear strengths of $\mathrm{C}_{60}$ clathrate along the (110)[001], (110)[1-10] and (110)[1-11] slip systems. Furthermore, the (100)[001] and (111)[11-2] slip systems were also considered. We obtained the critical shear strengths of 90.3, 76.4, 89.2, 91.8, and 93.3 GPa for (110)[001], (110)[1-10], (110)[1-11], (100)[001], and (111)[11-2] slip systems, respectively (Fig. 4c). Consequently, the lowest shear strength of $\mathrm{C}_{60}$ clathrate is $76.4 \mathrm{GPa}$, less than its tensile strength, suggesting that the crystal is more sensitive to the shear deformation. The close value of shear strength and hardness of $\mathrm{C}_{60}$ clathrate is due to the lowest shear strength that almost reflects the real hardness of the material. 

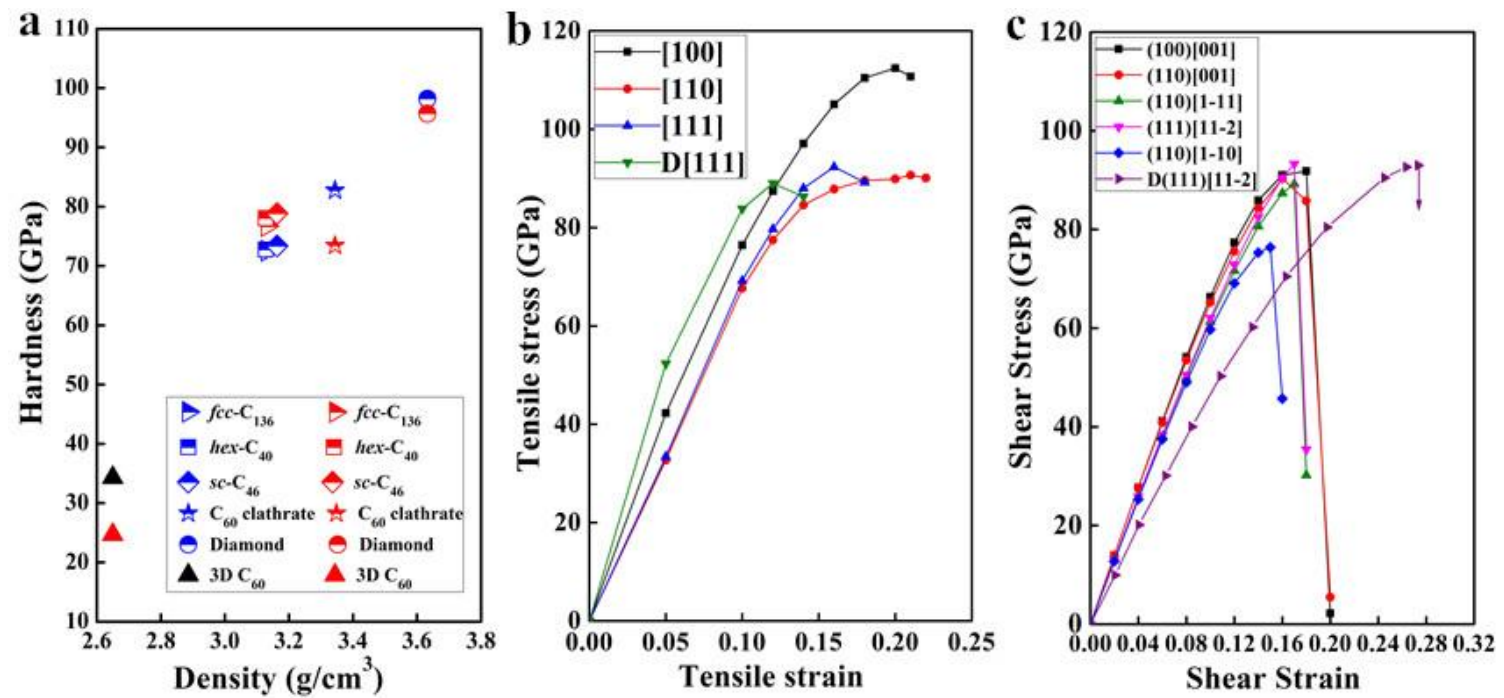

Figure 4. Hardness and densities of $\mathrm{C}_{60}$ clathrate and other carbon allotropes (a), the ideal tensile strengths of $\mathrm{C}_{60}$ clathrate along [100], [110] and [111] directions (b), and the ideal shear strengths of $\mathrm{C}_{60}$ clathrate along various directions (c). In Fig.4a, the hardness values estimated from Gao's and Chen's models are marked as blue and red color, respectively. The experimental data of $3 \mathrm{D} \mathrm{C}_{60}$ is labeled as the black symbol. In Fig. $4 \mathrm{~b}$ and $4 \mathrm{c}$, the $\mathrm{D}[111]$ and $\mathrm{D}(111)[11-2]$ represent the weakest crystal orientations of diamond during tension and shear deformation $[55,57]$. 


\section{Conclusions}

In summary, we have proposed predicted a novel $s p^{3}$-hybridized carbon clathrate, namely, $\mathrm{C}_{60}$ clathrate. $\mathrm{C}_{60}$ clathrate is a superdense, superhard, superstrong carbon clathrate with mechanical performance comparable with that of diamond. $\mathrm{C}_{60}$ clathrate is also a direct band gap semiconductor, potentially showing efficient light absorption and emission. Despite its high density, $\mathrm{C}_{60}$ clathrate can still adopt some small guest atoms, such as $\mathrm{Li}$ and $\mathrm{Na}$, in the $\mathrm{C}_{24}$ cages; it may become a superconductor like the recently discovered alkali metal-doped fullerene cages with high superconducting transition temperatures [58]. Our current research can stimulate the further exploration of novel carbon clathrates with attractive mechanical and electronic properties.

\section{Acknowledgements}

This work was supported by the National Science Foundation of China (Grants Nos. 51421091, 51332005, and 51272227), the Natural Science Foundation for Distinguished Young Scholars of Hebei Province of China (Grant No. E2014203150), and the Postgraduate Innovation Project of Hebei Province of China (Grant No. 00302-6370007).

\section{References}

[1] Heimann R, Evsvukov S, Koga Y. Carbon allotropes: a suggested classification scheme based on valence orbital hybridization. Carbon. 1997;35(10):1654-8.

[2] lijima S. Helical microtubules of graphitic carbon. Nature. 1991;354(6348):56-8.

[3] Ekimov E, Sidorov V, Bauer E, Mel'Nik N, Curro N, Thompson J, et al. Superconductivity in diamond. Nature. 2004;428(6982):542-5.

[4] Meyer JC, Geim AK, Katsnelson M, Novoselov K, Booth T, Roth S. The structure of suspended graphene sheets. Nature. 2007;446(7131):60-3.

[5] Wang X, Scandolo S, Car R. Carbon Phase Diagram fromAb InitioMolecular Dynamics. Physical Review Letters. 2005;95(18).

[6] Benedek G, Galvani E, Sanguinetti S, Serra S. Hallow diamonds: stability and elastic properties. Chemical Physics Letters. 1995;244(5-6):339-44.

[7] Herrmann RF, Tanigaki K, Kawaguchi T, Kuroshima S, Zhou O. Electronic structure of Si and Ge gold-doped clathrates. Physical Review B. 1999;60(19):13245.

[8] Kasper JS, Hagenmuller P, Pouchard M, Cros C. Clathrate structure of silicon $\mathrm{Na}_{8} \mathrm{Si}_{46}$ and $\mathrm{Na}_{x} \mathrm{Si}_{136}$ (x<11). Science. 1965;150(3704):1713-4.

[9] San-Miguel A. Nanomaterials under high-pressure. Chemical Society reviews. 
2006;35(10):876-89.

[10] Guloy AM, Ramlau R, Tang Z, Schnelle W, Baitinger M, Grin Y. A guest-free germanium clathrate. Nature. 2006;443(7109):320-3.

[11] Ramachandran GK, Dong J, Diefenbacher J, Gryko J, Marzke RF, Sankey OF, et al. Synthesis and X-ray characterization of silicon clathrates. Journal of Solid State Chemistry. 1999;145(2):716-30.

[12] San-Miguel A, Kéghélian P, Blase X, Mélinon P, Perez A, Itié J, et al. High pressure behavior of silicon clathrates: a new class of low compressibility materials. Physical Review Letters. 1999;83(25):5290.

[13] Beekman M, Baitinger M, Borrmann H, Schnelle W, Meier K, Nolas GS, et al. Preparation and crystal growth of $\mathrm{Na}_{24} \mathrm{Si}_{136}$. Journal of the American Chemical Society. 2009;131(28):9642-3.

[14] Adams GB, O'Keeffe M, Demkov AA, Sankey OF, Huang Y-M. Wide-band-gap Si in open fourfold-coordinated clathrate structures. Physical Review B. 1994;49(12):8048-53.

[15] Rey N, Muñoz A, Rodríguez-Hernández P, San Miguel A. First-principles study of lithium-doped carbon clathrates under pressure. Journal of Physics: Condensed Matter. 2008;20(21):215218.

[16] Blase X. Quasiparticle band structure and screening in silicon and carbon clathrates. Physical Review B. 2003;67(3).

[17] Blase X, Gillet P, San Miguel A, Melinon P. Exceptional ideal strength of carbon clathrates. Phys Rev Lett. 2004;92(21):215505.

[18] Ribeiro FJ, Tangney P, Louie SG, Cohen ML. Hypothetical hard structures of carbon with cubic symmetry. Physical Review B. 2006;74(17).

[19] Li D, Tian F, Chu B, Duan D, Wei S, Lv Y, et al. Cubic $C_{96}$ : a novel carbon allotrope with a porous nanocube network. J Mater Chem A. 2015;3(19):10448-52.

[20] Hu M, Tian F, Zhao Z, Huang Q, Xu B, Wang L-M, et al. Exotic Cubic Carbon Allotropes. The Journal of Physical Chemistry C. 2012;116(45):24233-8.

[21] Winkler B, Milman V. Structure and properties of supercubane from density functional calculations. Chemical physics letters. 1998;293(3):284-8.

[22] Gal'pern E, Sabirov AR, Stankevich I, Chistyakov A, Chernozatonskii LA. A new crystalline form of carbon based on the $C_{36}$ fullerene: Simulating its crystal and electronic structure. Journal of Experimental and Theoretical Physics Letters. 2001;73(9):491-4.

[23] Popov A, Bazhin I. CUBIC POlyMerized StRUCTUReS OF SMALl FUllerenes $C_{20}, C_{24}, C_{28}, C_{32}$. Hydrogen Materials Science and Chemistry of Carbon Nanomaterials: Springer; 2007. p. 713-9.

[24] Adams GB, Sankey OF, Page JB, O'Keeffe M. Jahn-Teller distortions in solid $C_{20}$ and other fullerene structures. Chemical physics. 1993;176(1):61-6.

[25] de Corato M, Proserpio DM, Bernasconi M, Benedek G. Two $\mathrm{C}_{28}$ Clathrates. Diamond and Related Nanostructures: Springer; 2013. p. 75-89.

[26] Yamanaka S, Kubo A, Inumaru K, Komaguchi K, Kini NS, Inoue T, et al. Electron conductive three-dimensional polymer of cuboidal $C_{60}$. Phys Rev Lett. 2006;96(7):076602.

[27] Yamanaka S, Kini NS, Kubo A, Jida S, Kuramoto $\mathrm{H}$. Topochemical 3D polymerization of $\mathrm{C}_{60}$ under high pressure at elevated temperatures. Journal of the American Chemical Society. 2008;130(13):4303-9.

[28] Wang Y, Lv J, Zhu L, Ma Y. Crystal structure prediction via particle-swarm optimization. Physical Review B. 2010;82(9).

[29] Segall M, Lindan PJ, Probert Ma, Pickard C, Hasnip P, Clark S, et al. First-principles simulation: ideas, illustrations and the CASTEP code. Journal of Physics: Condensed Matter. 2002;14(11):2717. 
[30] Kohn W, Sham LJ. Self-Consistent Equations Including Exchange and Correlation Effects. Physical Review. 1965;140(4A):A1133-A8.

[31] Hohenberg P, Kohn W. Inhomogeneous Electron Gas. Physical Review. 1964;136(3B):B864-B71.

[32] Jones RO, Gunnarsson O. The density functional formalism, its applications and prospects. Reviews of Modern Physics. 1989;61(3):689-746.

[33] Monkhorst HJ, Pack JD. Special points for Brillouin-zone integrations. Physical Review B. 1976;13(12):5188-92.

[34] Fischer TH, Almlof J. General methods for geometry and wave function optimization. The Journal of Physical Chemistry. 1992;96(24):9768-74.

[35] Clark SJ, Segall MD, Pickard CJ, Hasnip PJ, Probert MIJ, Refson K, et al. First principles methods using CASTEP. Zeitschrift für Kristallographie. 2005;220(5/6/2005).

[36] Zhang $\mathrm{Y}$, Sun $\mathrm{H}$, Chen C. Superhard cubic $\mathrm{BC}_{2} \mathrm{~N}$ compared to diamond. Phys Rev Lett. 2004;93(19):195504.

[37] Roundy D, Krenn C, Cohen ML, Morris Jr J. Ideal shear strengths of fcc aluminum and copper. Physical Review Letters. 1999;82(13):2713.

[38] Amsler M, Flores-Livas JA, Lehtovaara L, Balima F, Ghasemi SA, Machon D, et al. Crystal Structure of Cold Compressed Graphite. Physical Review Letters. 2012;108(6).

[39] Li Q, Ma Y, Oganov AR, Wang H, Wang H, Xu Y, et al. Superhard Monoclinic Polymorph of Carbon. Physical Review Letters. 2009;102(17).

[40] Qingkun L, Yi S, Zhiyuan L, Yu Z. Lonsdaleite - A material stronger and stiffer than diamond. Scripta Materialia. 2011;65(3):229-32.

[41] Wang J-T, Chen C, Kawazoe Y. Low-Temperature Phase Transformation from Graphite to $\mathrm{sp}^{3}$ Orthorhombic Carbon. Physical Review Letters. 2011;106(7).

[42] Zhao Z, Xu B, Zhou X-F, Wang L-M, Wen B, He J, et al. Novel Superhard Carbon: C-Centered Orthorhombic $C_{8}$. Physical Review Letters. 2011;107(21).

[43] Hu M, Pan Y, Luo K, He J, Yu D, Xu B. Three dimensional graphdiyne polymers with tunable band gaps. Carbon. 2015;91:518-26.

[44] the Reticular Chemistry Structure Resource (RCSR) database http://rcsr.net/nets/sdt.

[45] Öhrström L, O'Keeffe M. Network topology approach to new allotropes of the group 14 elements. Zeitschrift für Kristallographie - Crystalline Materials. 2013;228(7):343-6.

[46] Wu Z-j, Zhao E-j, Xiang H-p, Hao X-f, Liu X-j, Meng J. Crystal structures and elastic properties of superhard IrN $\mathrm{N}_{2}$ and IrN $\mathrm{N}_{3}$ from first principles. Physical Review B. 2007;76(5).

[47] Pugh SF. XCII. Relations between the elastic moduli and the plastic properties of polycrystalline pure metals. The London, Edinburgh, and Dublin Philosophical Magazine and Journal of Science. 2009;45(367):823-43.

[48] Hill R. The elastic behaviour of a crystalline aggregate. Proceedings of the Physical Society Section A. 1952;65(5):349.

[49] Frantsevich I, Voronov F, Bokuta S, Frantsevich I. Elastic constants and elastic moduli of metals and insulators handbook. Naukova Dumka, Kiev. 1983:60-180.

[50] Gao F, He J, Wu E, Liu S, Yu D, Li D, et al. Hardness of Covalent Crystals. Physical Review Letters. 2003;91(1).

[51] Phillips JC. Ionicity of the Chemical Bond in Crystals. Reviews of Modern Physics. 1970;42(3):317-56.

[52] Niu H, Chen XQ, Wang S, Li D, Mao WL, Li Y. Families of superhard crystalline carbon allotropes 
constructed via cold compression of graphite and nanotubes. Phys Rev Lett. 2012;108(13):135501.

[53] Chen X-Q, Niu H, Franchini C, Li D, Li Y. Hardness of T-carbon: Density functional theory calculations. Physical Review B. 2011;84(12).

[54] Chen X-Q, Niu H, Li D, Li Y. Modeling hardness of polycrystalline materials and bulk metallic glasses. Intermetallics. 2011;19(9):1275-81.

[55] Zhou X-F, Qian G-R, Dong X, Zhang L, Tian Y, Wang H-T. Ab initiostudy of the formation of transparent carbon under pressure. Physical Review B. 2010;82(13).

[56] Pan Z, Sun H, Zhang Y, Chen C. Harder than diamond: superior indentation strength of wurtzite BN and lonsdaleite. Phys Rev Lett. 2009;102(5):055503.

[57] Telling R, Pickard C, Payne M, Field J. Theoretical strength and cleavage of diamond. Physical Review Letters. 2000;84(22):5160.

[58] Spagnolatti I, Bernasconi M, Benedek G. Electron-phonon interaction in the solid form of the smallest fullerene $C_{20}$. EPL (Europhysics Letters). 2002;59(4):572. 